\section{Development, Detection, and Elimination of Verticillium dahliae in Mint Shoot Cultures}

\author{
Nan Wang \\ Department of Horticulture, Oregon State University, Corvallis, OR 97331 \\ Barbara M. Reed ${ }^{1}$ \\ USDA-ARS, National Clonal Germplasm Repository, 33447 Peoria Road, \\ Corvallis, OR 97333-2521
}

Additional index words. certification, meristem culture, Mentha, peppermint, spearmint, Verticillium wilt

\begin{abstract}
Roots of greenhouse-grown mint plants and in-vitro-grown shoot cultures were inoculated with Verticillium dahliae Kleb. conidial suspensions to study wilt symptom development and detection and elimination of the fungus. There were significant differences in the symptom expression between control and infected shoot cultures at all conidia concentrations for the four mints tested. Disease-symptom ratings were proportional to the $V$. dahliae inoculum density. Infected shoot cultures were stunted when inoculated with $\geq 10^{3}$ conidia/mL. Verticillium dahliae was re-isolated from infected shoot cultures at all levels of inoculum, but not from any control cultures. Verticillium infections were easily detected by plating mint stems on potato dextrose agar. Shoot tips $(0.5$ to $15 \mathrm{~mm})$ from infected in-vitro- and greenhouse-grown plants were isolated and screened for fungus. The most effective shoot length for fungus elimination was 3-5 $\mathbf{~ m m}$. Shoot tips isolated from in vitro spearmint cultivars infected at $10^{2}$ and $10^{3}$ conidia/mL were $100 \%$ Verticillium free, but only $42 \%$ of 'Black Mitcham' and 54\% of 'Todd's Mitcham' peppermints were free of the disease. Shoot tips from infected greenhouse plants produced Verticillium-free cultures from $79 \%$ of 'Black Mitcham' and $90 \%$ of 'Todd's Mitcham' plants. These results indicate the utility of testing for Verticillium and the safety of micropropagated mint shoots for certified planting stock programs.
\end{abstract}

Verticillium dahliae Kleb. (=V.albo-atrum var. menthae Nelson), a soilborne vascular wilt pathogen, is economically important in mint production. Most commercial mint cultivars are susceptible, and vegetative propagation of mint by rootstocks and runners is responsible for its distribution in North America. (Brandt et al., 1984; Sink and Grey, 1999).

Maintenance of specific clonally propagated genotypes of spearmint and peppermint is highly important to the mint-oil industry. Certification of stock for field planting is currently valid only for plants derived from certified mother-blocks. Plant tissue culture is used for distributing germplasm of many clonally propagated crops. Micropropagation of mint would provide a convenient method for distributing certified Verticillium-free mint plants. Although cultures can be tested for the presence of viruses, no method is available to certify that in-vitro-grown plants are free of fungi. It is important to determine if standard laboratory procedures for detecting Verticillium

\footnotetext{
Received for publication 28 Jan. 2002. Accepted for publication 19 June 2002. This research was supported by ARS CRIS 5853-21000-026-00D and a grant from the A.M. Todd Co. to B. Reed. Use of trade names in this publication does not imply endorsement of the U.S. Dept. of Agriculture (USDA) or Oregon State Univ.

${ }^{1}$ To whom requests for reprints should be addressed. E-mail address: reedbm@bcc.orst.edu
}

are effective for in vitro cultures and can be used to certify mint cultures as Verticillium free. Symptoms of Verticillium wilt disease on Mentha field plants are widely described (Baker, 1981; Nelson, 1950). However, there are no published reports on the expression of wilt symptoms of in-vitro-grown mint plants.

The objectives of this research were to 1 ) determine if standard plant pathology procedures are effective for detecting $V$.dahliae infections of in vitro cultures; 2 ) if plating techniques can be used to certify mint cultures as Verticillium free; 3) to develop an effective method to eliminate Verticillium from infected plants.

\section{Materials and Methods}

In vitro plants. Native spearmint, Mentha spicata L. (Local identifier: Men 582.001); scotch spearmint, Mentha $\times$ gracilis Sole (Men 583.001); and peppermint, Mentha $\times$ piperita L. cvs. 'Black Mitcham' (Men 579.001), and 'Todd's Mitcham' (Men 581.001) were propagated from shoot cultures held at the National Clonal Germplasm Repository (NCGR) in Corvallis, Ore. Shoots were multiplied in Magenta GA7 vessels (Magenta Corp., Chicago) containing $40 \mathrm{~mL}$ MS medium (Murashige and Skoog, 1962) with $2.2 \mathrm{~mm} N^{6}$-benzyladenine (BA) and $0.5 \mathrm{~mm}$ indole-3-butyric acid (IBA), 3\% sucrose, $0.3 \%$ Bitek agar (Difco, Detroit), and $0.125 \%$ Gelrite (Schweitzer-Hall, South Plainfield, N.J.) at pH 5.7. Shoot tips $(2 \mathrm{~cm})$ with two nodes were harvested from 3-weekold shoot cultures and rooted for $6 \mathrm{~d}$ in Magenta vessels containing $10 \mathrm{mLMS}$ medium without plant growth regulators (rooting medium).

Greenhouse plants. Shoots were collected from pot-grown screenhouse plants of the same accessions and propagated in $3 \times 5 \times$ $5-\mathrm{cm}$ pots with 12 pots for each genotype and at least 10 pieces $(3-5 \mathrm{~cm})$ in each pot. The lower leaves were removed and the base of the stem was inserted $\approx 2 \mathrm{~cm}$ in sterile medium (1 sphagnum peat : 1 pumice : 1 fir fines). Plants were repotted as needed in order to maintain actively growing, nonflowering plants.

Inoculum. A stock culture of $V$. dahliae was stored on potato dextrose agar (PDA) in a sealed tube at $5{ }^{\circ} \mathrm{C}$. Inoculum was grown on PDA at $23^{\circ} \mathrm{C}$. Conidial suspensions were prepared by scraping the culture surface with a loop and mixing the conidia with sterile deionized (DI) water (Sharma and Nowak, 1998). The suspension was added to sterile DI water, homogenized on a tube mixer (Vortex; American Hospital Supply, Evanston, Ill.) for $1 \mathrm{~min}$, and filtered through four layers of cheesecloth to remove mycelial fragments and microsclerotia. Conidial concentrations were estimated with a hemacytometer and adjusted to $1 \times 10^{6}$ conidia/mL using DI water.

Inoculation and pretesting for Verticillium infection, Leaves were removed from 8-10 in-vitro-grown shoots of each cultivar, the stems were cut into $3-5 \mathrm{~mm}$ segments and planted upright in petri dishes $(100 \times 15 \mathrm{~mm})$ containing $20 \mathrm{~mL}$ PDA for $5 \mathrm{~d}$. Stem sections and media were observed for the presence of fungi after 2 weeks.

In vitro infection of mint plantlets. Verticillium conidial suspensions of $10^{2}$ to $10^{6}$ and a DI water control were prepared and $1 \mathrm{~mL}$ dispensed into sterile tubes $(16 \times 100 \mathrm{~mm})$. Root tips were trimmed from in vitro plantlets of each cultivar and the roots submerged in the conidial suspensions. Plantlets remained in the suspension under light in the laminar-flow hood for $30 \mathrm{~min}$ before transfer to tubes $(16 \times 100$ $\mathrm{mm}$ ) of rooting medium. Ten plantlets were inoculated per treatment and the experiment was conducted twice $(n=20)$. Wilt symptoms, including stem length, stem thickness, foliar chlorosis, and percentage of fungus on stem pieces, were noted at 4 weeks. Stem diameter was measured 2 and $4 \mathrm{~cm}$ from the medium surface with electronic calipers (Max-Cal; Fowler, Japan). Symptoms of foliar chlorosis were rated on a scale of $0-4$, where $0=$ no chlorosis, $1=$ $1 \%-25 \%, 2=25 \%-50 \%, 3=50 \%-75 \%$, and 4 $=75 \%-100 \%$. Inoculated plantlets were tested using the same procedure as for pretesting. Data were recorded at 4 weeks.

Ex vivo infection of mint plants. A $10^{5}$ conidia/mL suspension of $V$. dahliae was prepared and $100 \mathrm{~mL}$ dispensed into $500-\mathrm{mL}$ plastic beakers. Roots were rinsed free of soil with running tap water, wounded slightly by trimming the root tips with a scalpel, and submerged in the conidial suspension or sterile DI water for $30 \mathrm{~min}$. The rooted cuttings were immediately planted and placed in a greenhouse at $25^{\circ} \mathrm{C}$ with a 14 -h light/10-h dark photoperiod. 
Testing for Verticillium infection of in vivo plants. Ten stem pieces were randomly collected from symptomatic and symptomless field-grown and greenhouse-grown plants. Leaves were removed and shoot tips rinsed with tap water for $15 \mathrm{~min}$ and surface disinfested in $10 \%$ commercial bleach $(5.25 \%$ sodium hypochlorite; Clorox, Oakland, Calif.) for $10 \mathrm{~min}$. Stems were cut into 3-5-mm segments and plated onto $20 \mathrm{~mL}$ streptomycin water agar medium $\left(100 \mathrm{~m} \cdot \mathrm{mL}^{-1}\right)$ in $100 \times$ 15-mm petri dishes.

Obtaining fungus-free cultures by shoot tip culture. Shoots (3-5 cm) of V.dahliae-infected greenhouse peppermint plants were harvested, the tips (1-2 cm) removed, surface disinfested in $10 \%$ bleach for $1 \mathrm{~min}$, and rinsed twice with sterile DI water. Shoot tips $(0.5-1 \mathrm{~mm}, 3-5$ $\mathrm{mm}$, and $10-15 \mathrm{~mm}$ ) were excised and plated in 24-cell plates (Costar, Corning N.Y.) with 2 $\mathrm{mL}$ of standard medium per well. In a second experiment, 3-5 mm shoot tips of in-vitrogrown ('Black Mitcham', 'Todd's Mitcham', native spearmint, and scotch spearmint) and field plants ('Black Mitcham') were excised from symptomatic and symptomless plants. All shoot tip cultures were transferred to tubes $(13 \times 100 \mathrm{~mm})$ of standard medium after 1 week. The experiment was conducted three times $(\mathrm{n}=12)$.

Statistical analysis. A completely randomized design was used for all experiments. Mint cultures were randomly selected from in-vitro-, greenhouse-, and field-grown plants and randomly assigned to each treatment. Statistical analysis was done using the SAS system (SAS Institute, 2000). Differences among group means were analyzed using analysis of variance and Fisher's Protected LSD test $(P=$ 0.05 ) and regression analysis.

\section{Results}

Verticillium detection in vitro. Tests of existing mint cultures found no fungal growth from any explants on PDAor water agar. Verticillium dahliae was re-isolated from the stems of all infected plants showing symptoms, whether in-vitro- or pot-grown plants. Known fungusfree cultures inoculated with the Verticillium conidia produced fungal growth on fungalculture medium (Fig. 1A) as well as tissueculture medium (Fig. 1B). A large amount of fungal growth was evident on tissue-culture medium of all infected cultures and made it very unlikely that Verticillium would spread unnoticed through plant tissue cultures.

Verticillium effects on in vitro plantlets. Regression analysis indicated that both inoculum density and cultivar (Fig. 2A) significantly affected leaf chlorosis ratings. The equation of the fitted model $=0.20+0.78 \cdot$ inoculum density $-0.03 \bullet$ inoculum density $\bullet$ cultivar. Most peppermint cultivars died when inoculated with $10^{6}$ conidia $/ \mathrm{mL}$, whereas spearmint remained alive and stems stayed green. 'Black Mitcham' was the most susceptible cultivar with significantly higher foliar chlorosis ratings at $10^{2}$ to $10^{5}$ conidia/mL than the other cultivars (Fig. 2A). Native spearmint, 'Todd's Mitcham', and scotch spearmint had lower foliar chlorosis

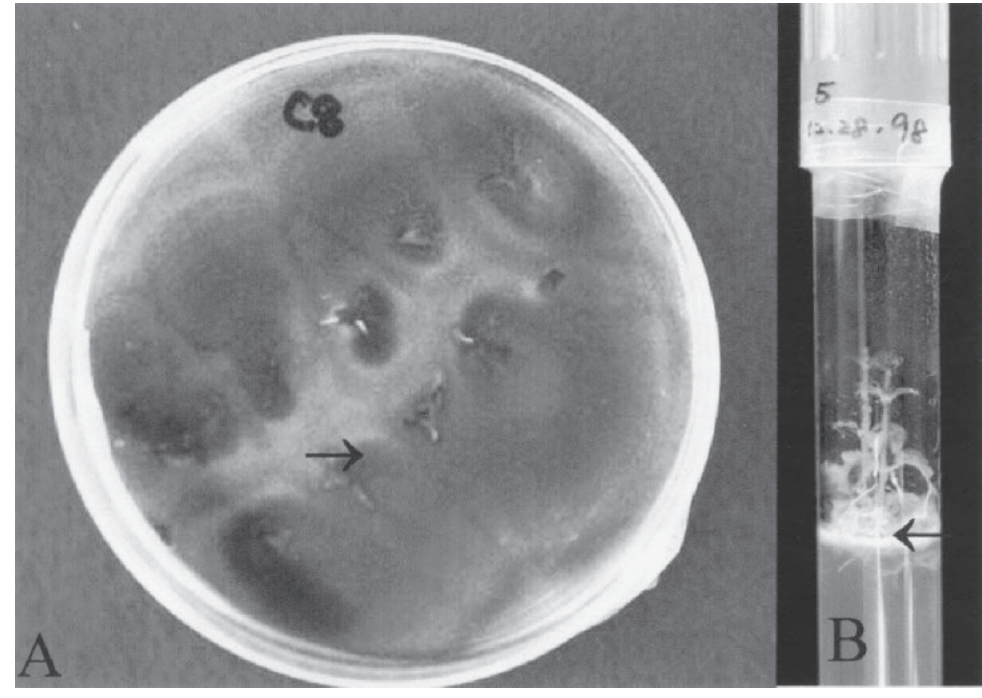

Fig. 1. Verticillium dahliae fungus growing on: (A) potato dextrose agar and (B) plant tissue culture medium.
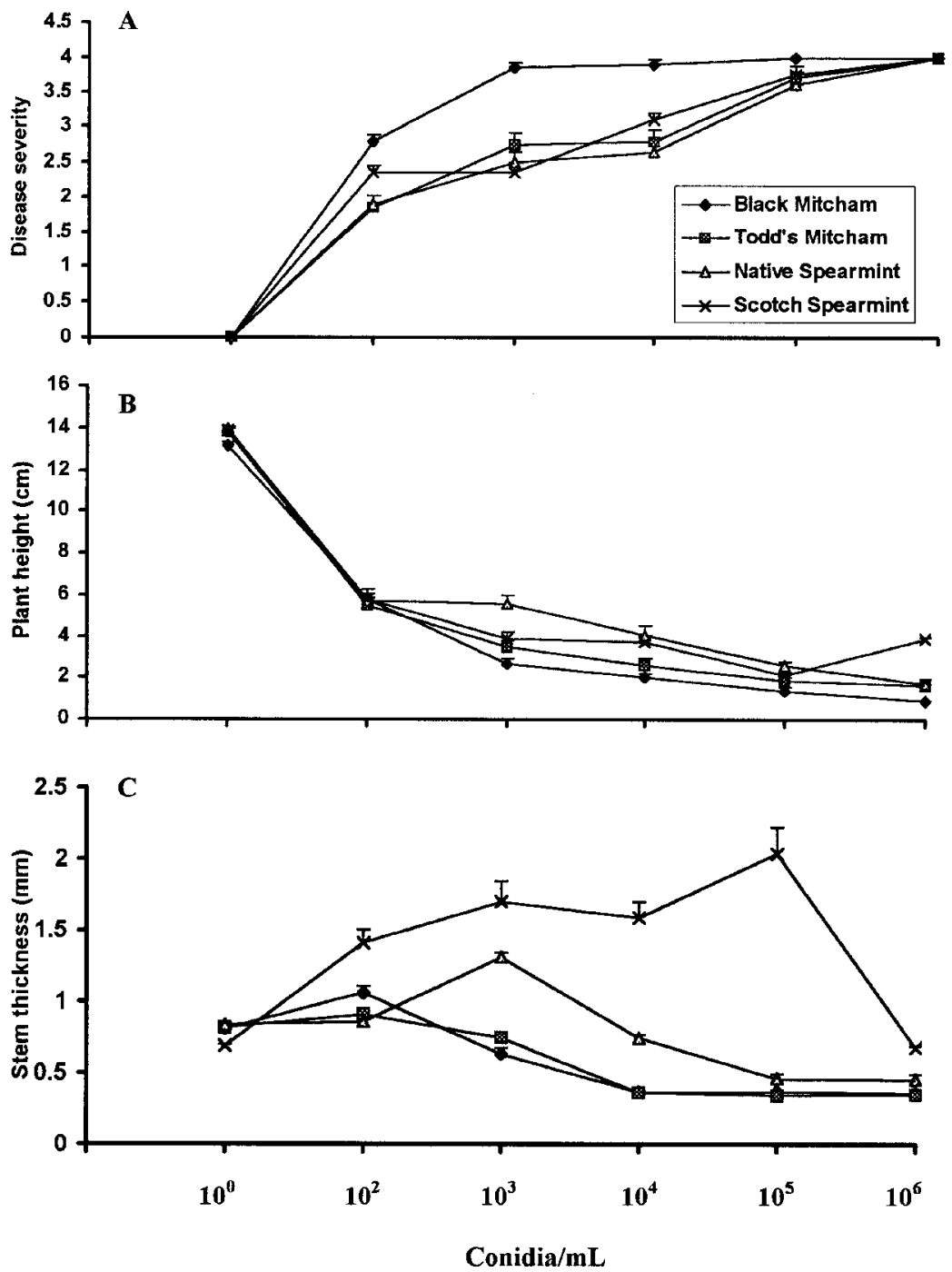

Fig. 2. In-vitro-grown 'Black Mitcham' peppermint, 'Todd's Mitcham' peppermint, native spearmint, and scotch spearmint infected with Verticillium dahliae spore suspensions at 0 (control), $10^{2}, 10^{3}, 10^{4}$, $10^{5}$, and $10^{6}$ conidia/mL. (A) Foliar chlorosis ratings $(0=$ no symptoms, $1=$ up to $25 \%$ of the foliage showing symptoms, $2=25 \%-50 \%, 3=75 \%, 4=75 \%-100 \%$ ). (B) Height increase of mint cultivars following in vitro infection. (C) Stem thickness at $4 \mathrm{~cm}$ above the medium surface following in vitro infection. Data were taken at 4 weeks $(n=10)$. Bars represent the standard error. 
ratings except at $10^{5}$ and $10^{6}$ conidia $/ \mathrm{mL}$. The shoot tips of all 'Todd's Mitcham' cultures died when inoculated with $10^{3}$ or more conidia/mL even though other parts of the plants were still green. All infected mint cultures were stunted. Significant differences $(P<0.05)$ in height were observed between the infected and noninfected cultures for all cultivars (Fig. 2B). All cultivars except native spearmint had an additional significant growth reduction at $10^{3}$ and $10^{4}$ conidia/mL. Shoot height (growth) decreased with increasing inoculum for all cultivars.

Significant differences in stem thickness were observed $(P \leq 0.05)$ between control and infected cultures at $10^{2}$ to $10^{5}$ conidia/mL for scotch spearmint (Fig. 2C). Stems were thicker than the controls and in plants inoculated at $10^{3}$ to $10^{5}$ conidia/mL stems thickened and split (Fig. $3 \mathrm{~A}$ and B). At $10^{6}$ conidia $/ \mathrm{mL}$ there was no significant difference between infected and control scotch spearmint cultures. Stems of 'Black Mitcham' and 'Todd's Mitcham' were significantly thinner $(P \leq 0.05)$ at $4 \mathrm{~cm}$ than the controls because of the severe wilt symptoms at $10^{3}$ to $10^{6}$ conidia/mL. Native spearmint stems were significantly thinner at $10^{4}$ to $10^{6}$ conidia/mL compared to the controls. Stem diameters $2 \mathrm{~cm}$ above the surface showed similar results (data not shown).

Effects of shoot tip size on production of Verticillium-free cultures. Shoot tip size and cultivar significantly affected the production of Verticillium-free cultures from infected greenhouse plants (Fig. 4A). The regression equation of the fitted model $=-1.46+1.39 \bullet$ treatment $+0.445 \bullet$ cultivar $-0.13 \bullet$ treatment - cultivar $-0.22 \bullet$ treatment $\bullet$ treatment. For the cultivars used in this experiment, shoot tips in the 3-5-mm range produced the most Verticillium-free cultures. Shoot tips in the two smallest size ranges (0.5-1 and $1-3 \mathrm{~mm})$ were often fungus free, but died from the excision process. Those in the largest size range (10-15 $\mathrm{mm}$ ) were initially alive, but many soon died from Verticillium infection. Shoot tip culture (3-5 mm) of infected greenhouse plants produced viable and Verticillium-free cultures from 79\% of 'Black Mitcham' and 96\% of 'Todd's Mitcham'.

Effects of initial inoculum and cultivar on the production of Verticillium-free shoot tips. Inoculum concentration and cultivar significantly affected the production of Verticilliumfree shoot tips from in-vitro-infected shoot cultures (Fig. 4B). The regression equation of the fitted model $=1.10-0.36 \bullet$ treatment + $0.078 \bullet$ treatment $\bullet$ cultivar. Verticillium-free cultures were obtained from all native and scotch spearmint shoots inoculated with $10^{2}$ and $10^{3}$ conidia $/ \mathrm{mL}$ while fewer were recovered at higher inoculum concentrations. Shoot-tip culture was only partially useful for peppermint with only $42 \%$ Verticillium-free cultures obtained for 'Black Mitcham' and 54\% for 'Todd's Mitcham' at $10^{2}$ conidia/mLand none at higher inoculum concentrations.

Testing of field plants for Verticillium infection and the production of Verticilliumfree shoot tips. Verticillium was isolated from shoot pieces of the field-grown plants of

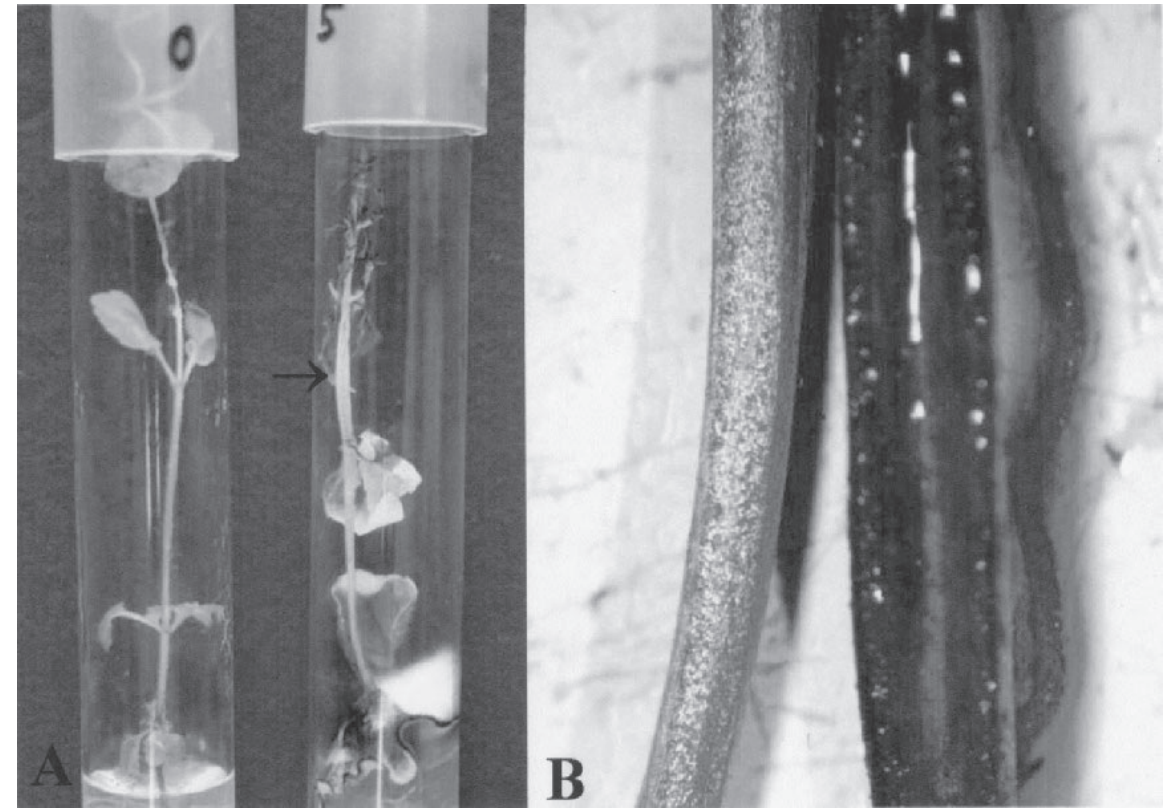

Fig. 3. (A) Scotch spearmint in vitro shoots: control (left) and inoculated with $10^{3} \sim 10^{5}$ conidia/mL showing split stems (right). (B) Enlarged view 40x: control (left) and inoculated stem (right).

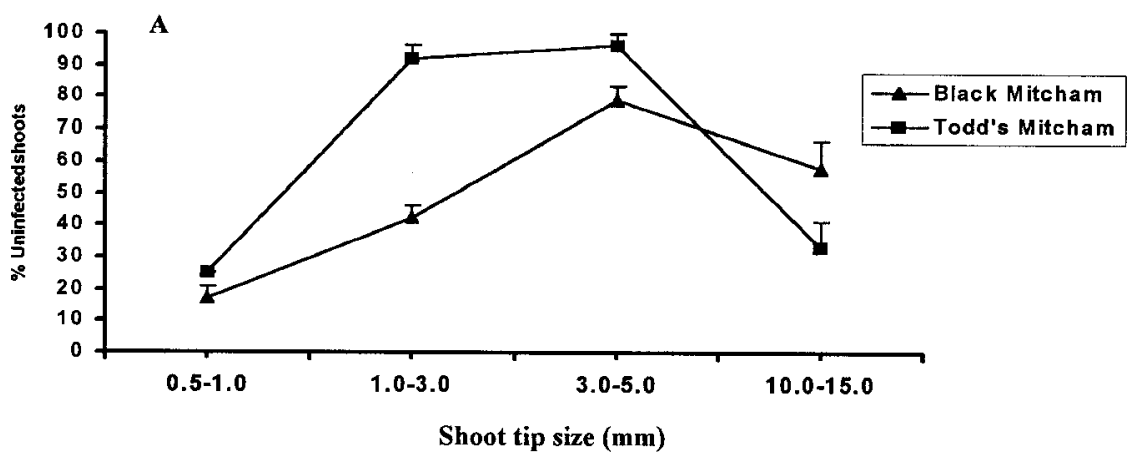

B

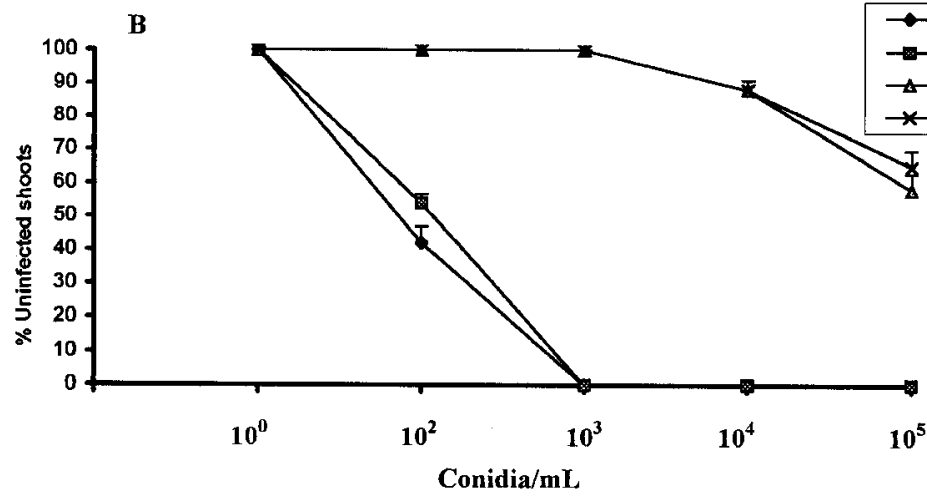

Fig. 4. (A) Percentage of Verticillium-free shoots produced from four size classes of excised shoot tips of in-vivo-infected greenhouse 'Black Mitcham' and 'Todd's Mitcham' peppermint inoculated with $10^{5}$ conidia/mL. (B) The percentage of Verticillium-free shoot tips 4 weeks after excision of 3-5-mm shoot tips from in-vitro-infected shoot cultures inoculated with Verticillium dahliae spore suspensions 0 (control), $10^{2}, 10^{3}, 10^{4}$, and $10^{5}$ conidia $/ \mathrm{mL}$. Bars represent the standard error.

'Black Mitcham' used for shoot tip excision. All shoot tips (3-5 mm) isolated and grown in vitro from infected field-grown plants were $100 \%$ free of Verticillium. Control plants were also Verticillium free.

\section{Discussion}

In-vitro-infected mint cultures exhibited symptoms similar to those of field-grown mint plants. However, the most typical diagnostic symptom in the field, unilateral development of young leaves (Baker, 1981) was not observed among in-vitro-infected plants. Symptoms varied among cultivars (Fig. 5A-C). Even though all the in-vitro-infected mint plantlets were stunted, shortening of the terminal internodes and smaller than normal terminal leaves were observed only on spearmint (Fig. $5 \mathrm{~A})$. The earliest symptom in peppermint was 


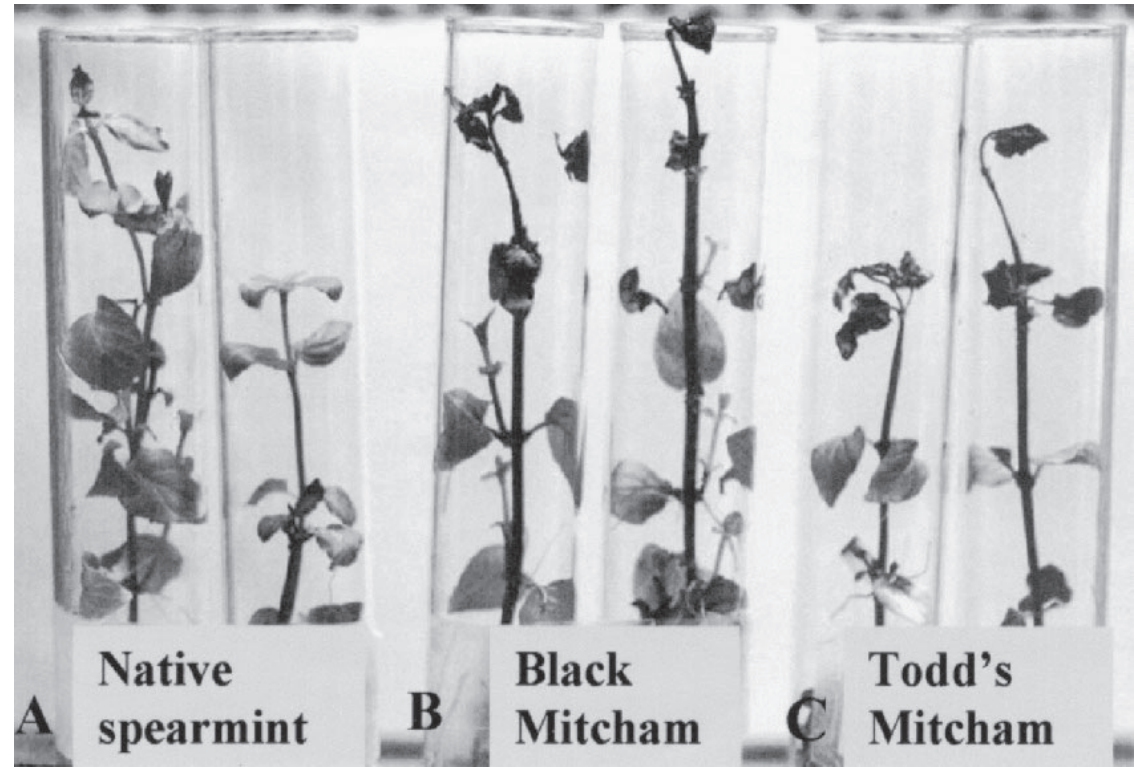

Fig. 5. Typical wilt symptoms of in-vitro-infected (A) native spearmint, (B) 'Black Mitcham', and (C) 'Todd's Mitcham' peppermint inoculated with $10^{3}$ conidia/mL.

wilting and discoloration of the stem (Fig. 5B-C). Wilting of spearmint shoot tips was observed late in the infection process.

Earlier studies show that vascular discoloration in the xylem and dark melanin pigments accumulated in host cells are caused by polyphenoloxidase that releases phenolic substrate from the infected plant tissues. The release of these pigments into the xylem can also lead to vascular occlusion (Arbogast, 1998; Green, 1981). 'Black Mitcham' is highly susceptible to Verticillium wilt and replacement cultivars were needed to improve mint production. 'Todd's Mitcham' was developed with moderate resistance to $V$. dahliae (Murray and Todd, 1975) and scotch spearmint (Mentha $\times$ gracilis Sole) was used as a substitute for peppermint because of its resistance to the fungus. Even though spearmints are also susceptible to wilt, they are less affected than peppermint in the field (Nelson, 1950; Sink and Grey, 1999). We observed similar results with in-vitro-grown mint plants. We noted that shoot tips in peppermint wilted much earlier than in spearmint (data not shown).

Hodgson and associates (1949) theorized that shoot tip culture would be useful for producing fungus-free mint cultures from infected stocks since the high molecular weight compounds that cause Verticillium wilting have less chance to reach the small vessels near the shoot tips (Hodgson et al., 1949). Shoot tip culture is used to obtain virus-free plants from infected stock and to produce pathogen-free plants from stock plants systematically infected with mycoplasma, fungi, and bacteria (Evans et al., 1983). Our study showed that it is possible to produce Verticillium-free plants from infected mother plants by shoot tip culture, even though the recovery percentage depends on the severity of the infection and the size of the shoot tip (Fig. 4). The more severe the infection and the smaller the shoot tip, the fewer viable and pathogen-free cultures were obtained. This process could be used to revitalize infected stock plots or to rescue important selections from Verticillium-infected fields.

This study determined that V. dahliae infection of mint cultures was easily detected by plating on a standard medium or by growth on the tissue-culture medium. Quantification of the disease process in vitro indicated that Verticillium-infected plants showed symptoms and died in culture within 3-6 weeks. The Verticillium fungus was never recovered from symptom-free plants. The severity of symptoms and the speed of plant death varied by cultivar with the peppermints more affected than the spearmints. Verticillium-infected plants from field, greenhouse, or in vitro could be freed of the fungus through excision and culture of 3-5-mm shoot tips. The effectiveness of shoot tip culture also depended on the initial inoculum density in the plant and the cultivar involved. The results of this study indicate the ease and utility of micropropagated mint shoots for distributing certified planting stock.

\section{Literature Cited}

Arbogast, M.C. 1998. Response of potato cultivars to moisture deficit stress and Verticillium dahliae. M.S. thesis, Oregon State Univ., Corvallis.

Baker, K.F. 1981. Biological Control, p. 549-557. In: M.E. Mace, A.A. Bell, and C.H. Beckman (eds.). Fungal wilt diseases of plants. Academic, New York.

Brandt, W.H., M.L. Lacy, and C.E. Horner. 1984. Distribution of Verticillium in stems of resistant and susceptible species of mint. Phytopathology 74:587-591.

Evans, D.A., W.R. Sharp, P.V. Ammirato, and Y. Yamada (eds.). 1983. Hdbk. of Plant Cell Culture, vol. 1. Macmillan, New York.

Green, R.J. 1981. An overview, p. 1-24. In: Fungal wilt disease of plants. Academic, New York.

Hodgson, T., W.H. Peterson, and A.J. Riker. 1949. The toxicity of polysaccharides and other large molecules to tomato cuttings. Phytopathology 39:47-62.

Murashige, T. and F. Skoog. 1962. A revised medium for rapid growth and bio assays with tobacco tissue cultures. Physiol. Plant. 15: 473-497.

Murray, M.J. and W.A. Todd. 1975. Registration of Todd's Mitcham peppermint. Crop Sci. 12:128.

Nelson, R. (ed.). 1950. Verticillium wilt of peppermint. Michigan State College, Agr. Expt. Sta., East Lansing, Mich.

SAS Institute. 2000. SAS/Graph user's guide, version 8.01. SAS Inst., Cary, N.C.

Sharma, V.K. and J. Nowak. 1998. Enhancement of Verticillium wilt resistance in tomato transplants by in-vitro co-culture of seedlings with a plant growth promoting rhizobacterium (Pseudomonas sp. strain PsJN). Can. J. Microbiol. 44:528-536.

Sink, K.C. and W.E. Grey. 1999. A root-injection method to assess Verticillium wilt resistance of peppermint (Mentha xpiperita L.) and its use in identifying resistant somaclones of $\mathrm{cv}$. Black Mitcham. Euphytica: Netherlands J. of Plant Breeding. 106:223-230. 